\title{
Broad-angle refractive transmodal elastic metasurface
}

Cite as: Appl. Phys. Lett. 117, 213502 (2020); https://doi.org/10.1063/5.0026928

Submitted: 26 August 2020 . Accepted: 09 November 2020 . Published Online: 23 November 2020

Sung Won Lee, (D) Hong Min Seung, (iD) Wonjae Choi, (iD) Miso Kim, and (iD) Joo Hwan Oh
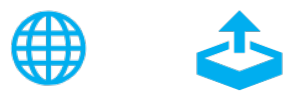

View Online

\section{ARTICLES YOU MAY BE INTERESTED IN}

Non-resonant metasurface for broadband elastic wave mode splitting

Applied Physics Letters 116, 171903 (2020); https://doi.org/10.1063/5.0005408

Amplitude-modulated binary acoustic metasurface for perfect anomalous refraction Applied Physics Letters 117, 221901 (2020); https://doi.org/10.1063/5.0032509

A compact low-frequency sound-absorbing metasurface constructed by resonator with embedded spiral neck

Applied Physics Letters 117, 221902 (2020); https://doi.org/10.1063/5.0031891

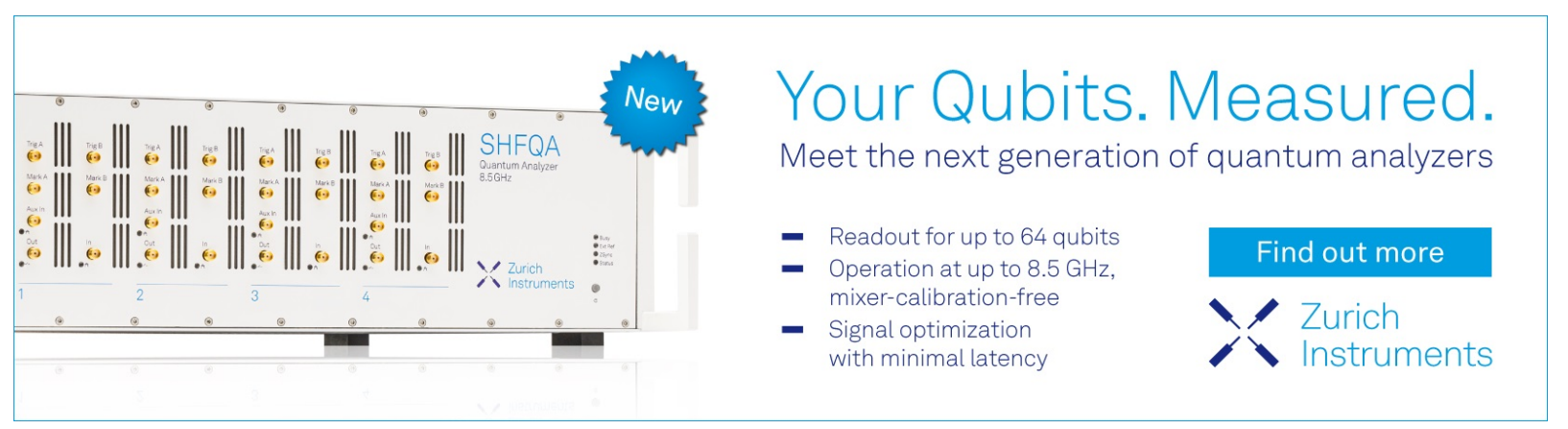




\title{
Broad-angle refractive transmodal elastic metasurface
}

\author{
Cite as: Appl. Phys. Lett. 117, 213502 (2020); doi: 10.1063/5.0026928 \\ Submitted: 26 August 2020 . Accepted: 9 November 2020 . \\ Published Online: 23 November 2020

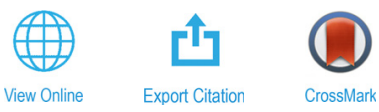

Sung Won Lee, ${ }^{7}$ Hong Min Seung, ${ }^{2}$ (D) Wonjae Choi, ${ }^{2}$ (D) Miso Kim, ${ }^{2}$ (D) and Joo Hwan Oh ${ }^{1, a)}$ (D)

\author{
AFFILIATIONS \\ 'School of Mechanical, Aerospace and Nuclear Engineering, Ulsan National Institute of Science and Technology, UNIST-gil 50, \\ Eonyang-eup, Ulju-gun, Ulsan 44919, South Korea \\ ${ }^{2}$ Al Metamaterial Research Team, Korean Research Institute of Standards and Science, Gajeong-ro 267, Yuseong-gu, \\ Daejeon 34113, South Korea
}

${ }^{a)}$ Author to whom correspondence should be addressed: joohwan.oh@unist.ac.kr. Tel.: +82-52-217-3051

\begin{abstract}
Achieving total mode conversion from longitudinal to shear waves for a broad incident angle has been a big scientific challenge in elastic fields, which was impossible to be achieved in classical elastic wave theory. In this paper, we propose and realize a refractive transmodal elastic metasurface that can convert an incident longitudinal wave to a shear wave for a broad incident angle. Here, the total mode conversion is achieved via a sufficiently large phase gradient, while the full transmission is achieved with the impedance-matched single-layered metasurface. Numerical and experimental investigations show that the proposed metasurface can provide almost total mode conversion for a broad incident angle from $-20.4^{\circ}$ to $22.3^{\circ}$. We expect that the proposed refractive transmodal metasurface can be applied in various ultrasonic systems.
\end{abstract}

Published under license by AIP Publishing. https://doi.org/10.1063/5.0026928

The existence of various modes such as longitudinal and shear waves is one of the unique characteristics that makes elastic waves different from other waves. ${ }^{1,2}$ Accordingly, the total mode conversion from the longitudinal to shear wave has attracted much attention due to not only its unique physical characteristics but also its fascinating possibilities in nondestructive evaluation (NDE) applications. ${ }^{3-6}$ However, the total mode conversion has been a tricky topic that could be achieved at a certain condition only. In classical elastic wave theory, the total mode conversion only takes place along a certain incident angle, which infers that unwanted longitudinal waves will come out with a slight angle difference. ${ }^{1,7}$ Recently, advances on metamaterials, ${ }^{8-14}$ artificial materials composed of sub-wavelength unit cells realizing unnatural properties such as negative parameters or uncommon wave characteristics, have opened a way for the total mode conversion. Wu et al. realized the negative shear modulus and density for the mode conversion from the shear wave to the longitudinal wave under a negative refractive angle. ${ }^{15}$ Zhu et al. realized the negative density and elastic modulus by translational and rotational resonances of chiral unit cells, leading to the mode conversion from the longitudinal to shear wave at a certain incident angle. ${ }^{16}$ Also, Zhu et al. theoretically and numerically studied the mode conversion ratio with various incident angles at double/triple negative media. ${ }^{17}$ Kweun et al. maximized mode conversion using transmodal Fabry-Pérot resonance at certain frequencies. ${ }^{18}$ Lee et al. proposed an elastic metamaterial wedge for perfect mode conversion from a longitudinal to shear wave at a certain incident angle and confirmed numerically and experimentally. ${ }^{19}$ Despite the recent research, however, the total mode conversion is still achievable with a certain incident angle.

Recently, there has been a breakthrough in achieving the total mode conversion by the idea of an elastic metasurface. A metasurface is an artificial thin layer that can control reflected or transmitted waves, ${ }^{20-27}$ such as broad band beam steering, ${ }^{28}$ wave absorption, ${ }^{29}$ and flexural wave steering. ${ }^{30,31} \mathrm{Kim}$ et al. proposed a way to achieve the total mode conversion for the broad incident angle, called the transmodal metasurface. ${ }^{32}$ Based on the generalized Snell's law, it was shown that the total mode conversion from the longitudinal to shear wave is possible for the broad incident angle, which is numerically and experimentally realized by the metasurface consisting of rod-like resonators. However, the previous research is based on the reflection-type metasurface; unlike the refraction-type metasurfaces utilizing refracted waves, reflection-type metasurfaces are hard to be further investigated for detailed physics and to be applied. It is obviously preferred to have the broad angle total mode conversion with a refraction-type metasurface.

In this work, we propose and realize a refraction-type metasurface realizing the total mode conversion for broad incident angles. 
Unlike in the previous research based on the reflection-type metasurface, there is a big scientific challenge that not only the total mode conversion but also the full transmission should be considered simultaneously. In general, the full transmission condition has been achieved via the Fabry-Pérot resonance inside the refraction-type metasurface, i.e., via designing the thickness of metasurface as a function of the wavelength. However, this approach is not valid in the current metasurface dealing with various incident angles where the wavevector component along the thickness direction is not constant, which infers that the Fabry-Pérot resonance cannot be used. Therefore, to realize the refraction-type transmodal metasurface with various incident angles, a full-transmission method, which is different from the Fabry-Pérot resonance, is essential.

Here, to overcome the challenge, the single-layered metasurface utilizing both positive and negative stiffness/mass values is introduced. $^{33}$ In the single-layered metasurface whose thickness is extremely shorter than the wavelength, wave transmission is dominated by the impedance, not by the Fabry-Pérot resonance. Thus, the single-layered metasurface can be tuned to achieve the full transmission at a broad incident angle. To achieve both the total mode conversion and the full transmission with a single-layered metasurface, first, the physics of the total mode conversion is theoretically investigated to show that the longitudinal wave with a broad incident angle can be converted to a shear wave only if the metasurface has an extremely large phase gradient. ${ }^{32}$ After then, the refractive transmodal elastic metasurface is proposed based on the single-layered metasurface. ${ }^{33}$ To examine the performance of the proposed refractive transmodal elastic metasurface, numerical and experimental validations will be presented.

The concept of the transmodal metasurface stems from the generalized Snell's law, ${ }^{34}$ which relates the refracted/reflected waves to the phase gradient of the metasurface. Assuming the full transmission, the refracted angle can be modulated by the phase gradient term as ${ }^{32}$

$$
k_{l} \sin \theta_{l}^{i}+\Psi=k_{l} \sin \theta_{l}^{r}=k_{s} \sin \theta_{s}^{r},
$$

where $k_{l}$ and $k_{s}$ are the longitudinal and shear wavevectors and $\Psi=\partial \phi / \partial x$ is the phase gradient term. The subscripts $l$ and $s$ refer to the longitudinal and shear properties, and the superscripts $i$ and $r$ refer to the incident or refracted wave properties, respectively. The generalized Snell's law, Eq. (1), infers that the refracted angles can be tailored by the phase gradient term, $\Psi$. Note that unlike the acoustics or electromagnetic waves, both the shear and longitudinal waves come out from the boundary with a single incident longitudinal wave due to the unique tensorial characteristics of the elastic wave.

Based on the generalized Snell's law, the total mode conversion is achieved by introducing a metasurface with sufficiently large phase gradient $\Psi$ so that $k_{l} \sin \theta_{l}^{i}+\Psi>k_{l}$ in Eq. (1). In this situation, the corresponding refracted angle for the longitudinal wave, $\theta_{l}^{r}$ in Eq. (1), becomes a complex number, indicating that the refracted longitudinal wave becomes the surface wave. (Note that this type of surface wave cannot exist without shear wave so that it cannot propagate through the outer region of the incident area. ${ }^{32}$ ) On the other hand, there exists a real-valued $\theta_{s}^{r}$ satisfying the generalized Snell's law so that only shear waves can be transmitted and refracted. As a result, only the shear wave exists for the transmitted field, i.e., the total mode conversion is available. $^{32}$ The total mode conversion takes place until the incident angle becomes large so that $k_{l} \sin \theta_{l}^{i}+\Psi=k_{s}$ is satisfied; if the incident angle becomes larger than this value, both longitudinal and shear waves are converted to the surface waves and the diffraction dominantly takes place. ${ }^{1,2}$ As a result, the total mode conversion can be achieved for the broad incident angle when $\theta_{l}^{c}<\theta_{l}^{i}<\theta_{s}^{c}$ as seen in Fig. 1, where $\theta_{l}^{c}$ and $\theta_{s}^{c}$ are defined as

$$
\begin{gathered}
\theta_{l}^{c}=\arcsin \left(1-\Psi / k_{l}\right), \\
\theta_{s}^{c}=\arcsin \left[\left(k_{s}-\Psi\right) / k_{l}\right] .
\end{gathered}
$$

In addition to the total mode conversion via the large phase gradient, the refractive transmodal elastic metasurface should have the full transmission characteristic, i.e., there should be almost no reflection from the metasurface. As explained earlier, the commonly applied idea based on the Fabry-Pérot resonance with multiple unit cells cannot be applied. Thus, we focus on the single-layered metasurface (the metasurface which has a single unit cell along the wave propagating direction), which can be simply represented with the mass-spring system shown in Fig. 2(a). In Fig. 2(a), it has been known that the full transmission can be achieved if ${ }^{33}$

$$
m_{e f f}=2 Z^{2} \alpha_{e f f} /\left(\alpha_{e f f}^{2}+\omega^{2} Z^{2}\right),
$$

and the corresponding phase shift is (the detailed derivations are given in the supplementary material)

$$
\phi=\operatorname{Arg}\left(\frac{\alpha_{e f f}{ }^{2}-\omega^{2} Z^{2}}{\alpha_{e f f}{ }^{2}+\omega^{2} Z^{2}}-\frac{2 \alpha_{e f f} Z \omega}{\alpha_{e f f}{ }^{2}+\omega^{2} Z^{2}} i\right) .
$$

Considering that the phase shift $\phi$ should vary from 0 to $2 \pi$ to apply the generalized Snell's law, the imaginary term in Eq. (4) should be varied from negative to positive values. Thus, the effective stiffness should be varied from negative to positive values, and accordingly, the effective mass should also be varied from negative to positive values by the impedance matching condition, Eq. (3). ${ }^{33}$ To realize such effective parameters, we use a unit cell consisting of vertical and horizontal resonators as shown in Fig. 2(b). It has been known that the horizontal and vertical resonators modulate the effective mass and stiffness from negative to positive values, respectively. ${ }^{10,33}$ (Also, the derivation of effective parameters from dual resonance can be seen in our supplementary material.) The detailed geometry of the unit cell is described in the supplementary material. By adjusting only heights $h_{1}$ and $h_{2}$ to modify the vertical and horizontal resonance states while the other

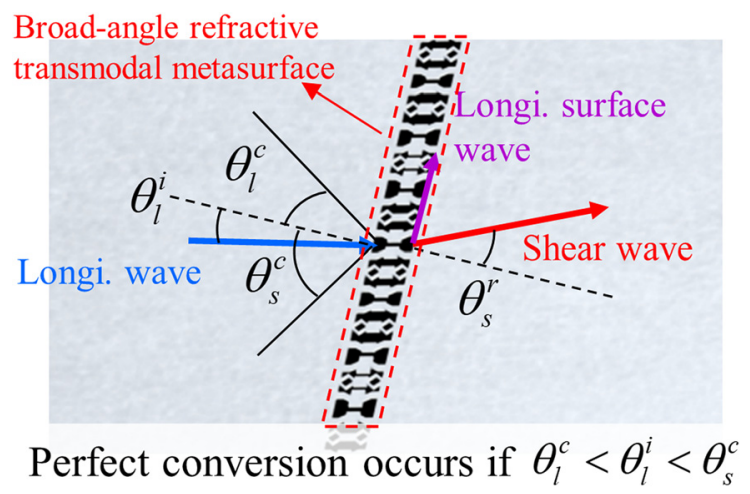

FIG. 1. Schematics of the broad-angle refractive transmodal metasurface. 
(a)

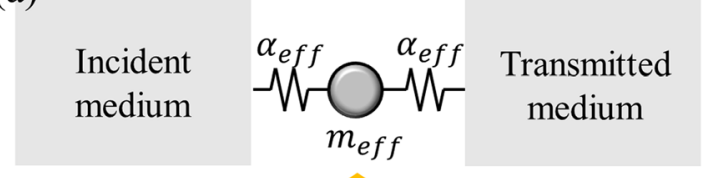

(b)

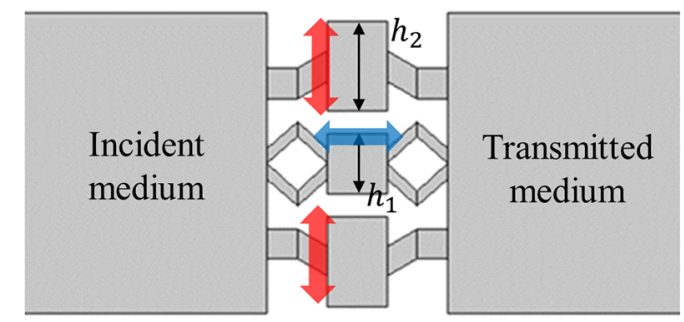

FIG. 2. (a) Mass-spring model and (b) the actual configuration for the singlelayered metasurface.

geometric parameters remain constant, one can tailor the effective mass and stiffness from negative to positive values. As a result, any phase gradient can be achieved with the full transmission.

Based on the metasurface unit shown in Fig. 2(b), the refractive transmodal elastic metasurface is designed. Here, the longitudinal wave incidence with the frequency of $50 \mathrm{kHz}$ is considered. To achieve the total mode conversion, four metasurface unit cells with the phase shift of $\phi=3 \pi / 8,7 \pi / 8,11 \pi / 8,15 \pi / 8$ are considered to achieve the phase gradient of $\Psi=25 \pi \mathrm{rad} / \mathrm{m}$. (Note that with the phase gradient, the total mode conversion is expected to take place at the incident angle from $-20.4^{\circ}$ to $22.3^{\circ}$ ). Figure 3 (a) plots the transmission coefficient and the phase shift for various values of $m_{1}$ and $m_{2}$. Based on the plots, 4 unit cells are designed with the desired phase shift and the full transmission as in Fig. 3(b). (The detailed values and the comparison between the analytically designed and numerically measured phase shifts are shown in the supplementary material.)

Based on the designed unit cells, various wave simulations are carried out to validate the total mode conversion of the proposed metasurface. Since the metasurface is designed to totally convert the longitudinal wave to shear wave for the incident angle from $-20.4^{\circ}$ to $22.3^{\circ}$, four incident angles of $-30^{\circ},-10^{\circ}, 0^{\circ}$, and $20^{\circ}$ are considered. To investigate the longitudinal and shear waves separately, the divergence and curl of displacements are plotted, respectively. Figure 4 plots the simulation results. As in Fig. 4(a), if the incident angle is $-30^{\circ}$, which is smaller than $\theta_{l}^{c}$, both the longitudinal and shear waves are refracted. However, if the incident angles are $-10^{\circ}, 0^{\circ}$, and $20^{\circ}$, which are between $\theta_{l}^{c}$ and $\theta_{s}^{c}$ as shown in Figs. 4(b)-4(d), the incident longitudinal wave is perfectly converted into the refracted shear wave and no refracted longitudinal wave is observed. To compare the numerically and analytically calculated refracted angles, black arrows are plotted along the analytically calculated directions in Fig. 4. Good agreement can be observed for all cases. As can be seen for all simulation results, it is validated that the proposed metasurface can almost fully convert the longitudinal wave to shear wave for broad incident angles with almost zero reflection.
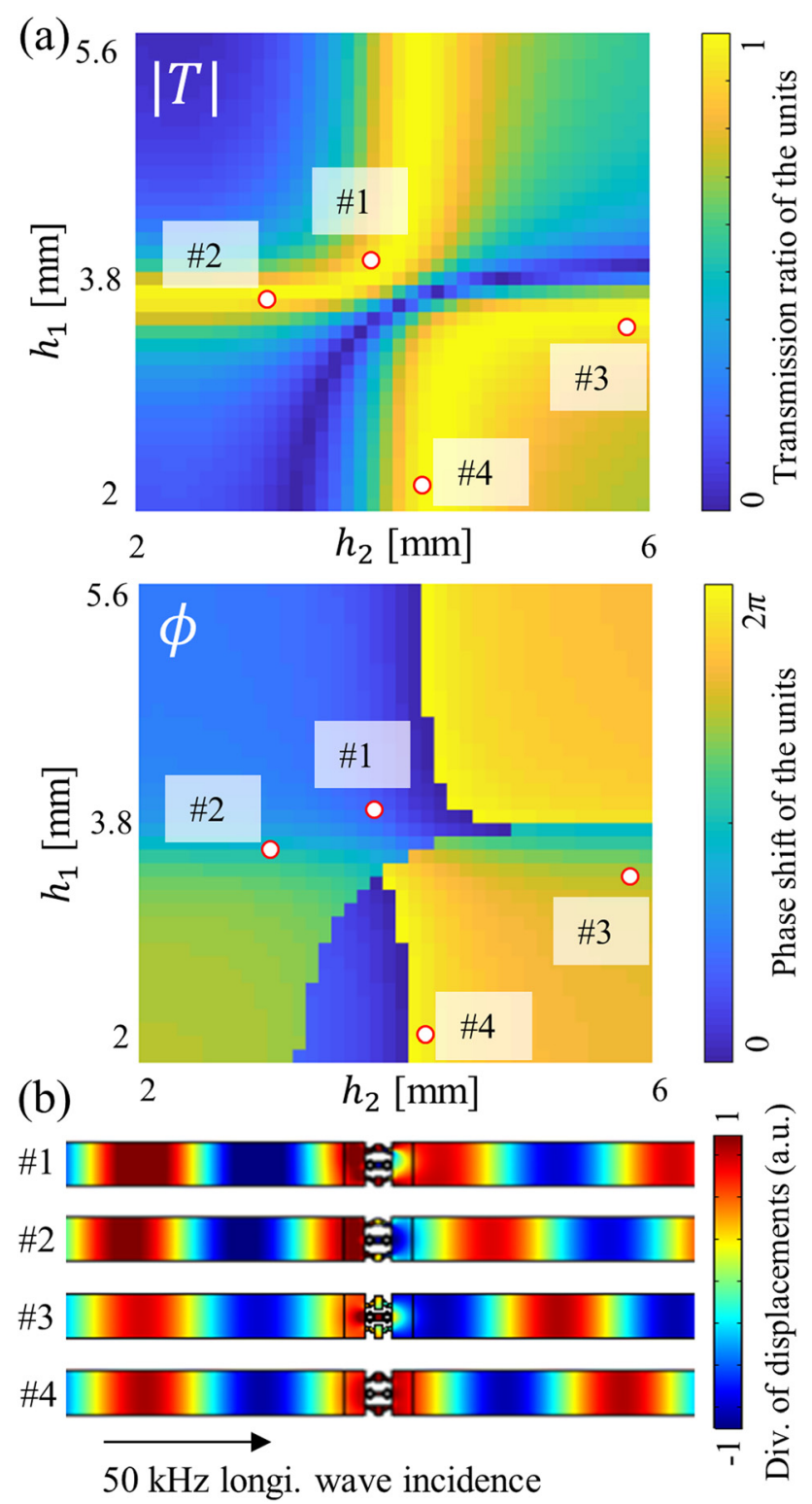

FIG. 3. (a) Plot of the transmission and phase shift for various $h_{1}$ and $h_{2}$. (b) Wave simulation for the designed 4 unit cells.

Finally, the experimental realization of the proposed refractive transmodal elastic metasurface is carried out. (The detailed experimental settings and process are given in the supplementary material.) Figure 5 shows the experimentally and numerically measured normalized amplitude of the refracted longitudinal and shear waves for various incident angles. Note that the simulations are carried out under the time-harmonic assumption, while the experiments are carried out with the transient pulse-type actuations. Nevertheless, very good agreement can be observed for all cases. For the incident angles of $-10^{\circ}, 0^{\circ}$, and $20^{\circ}$, almost no refracted longitudinal waves are measured compared to the shear waves. This strongly supports that the 
(a)
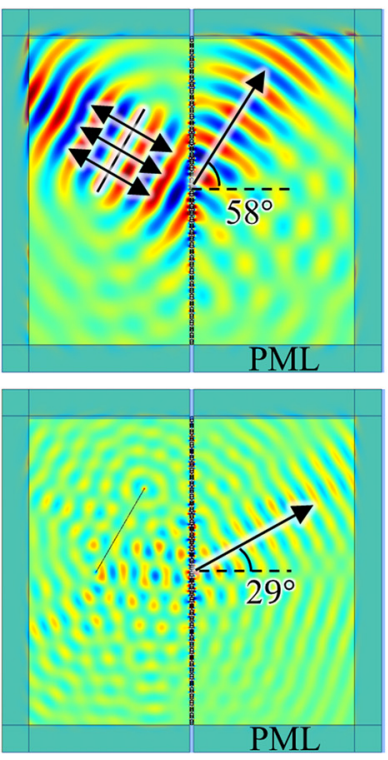

(b)
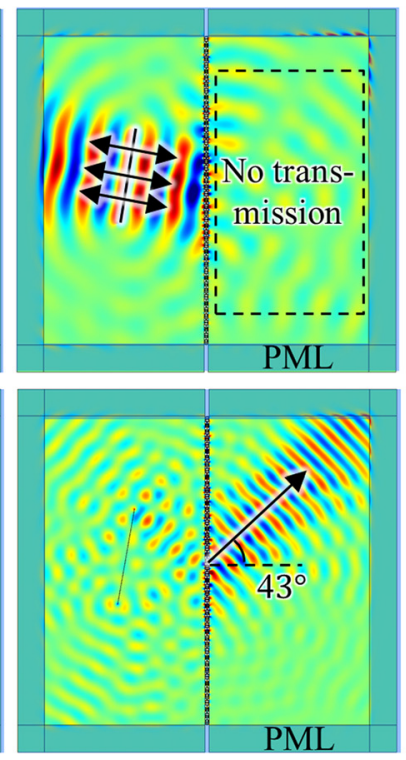

(c)
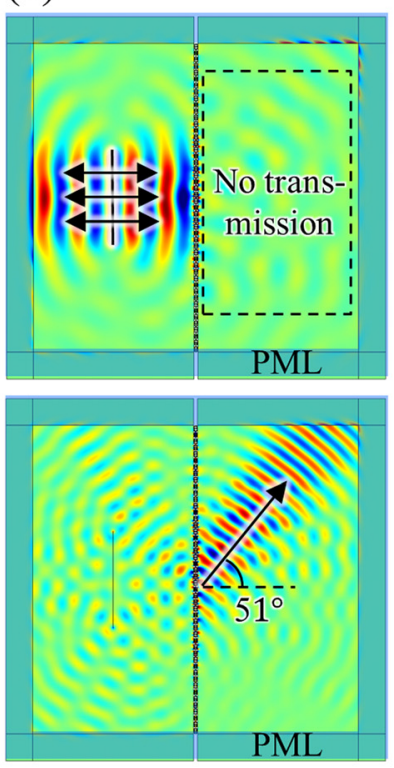

(d)
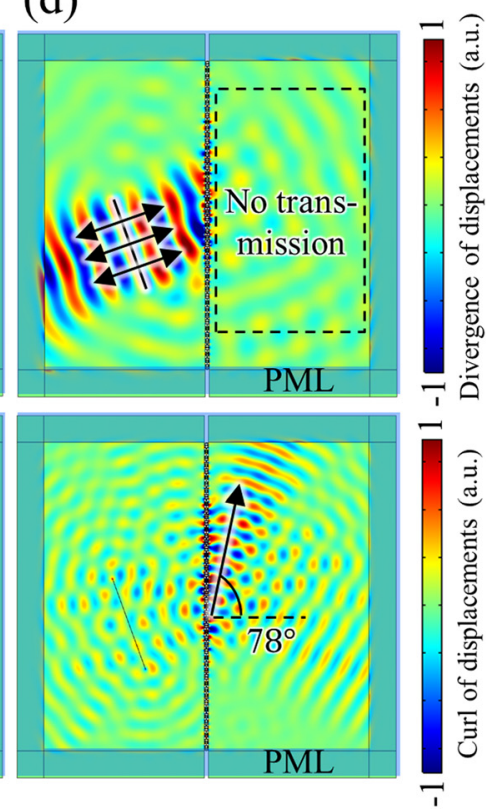

FIG. 4. Simulation results of the longitudinal wave with an incident angle of (a) $-30^{\circ}$, (b) $-10^{\circ}$, (c) $0^{\circ}$, and (d) $20^{\circ}$, respectively. The upper side figures correspond to the longitudinal fields, while the lower sides, the shear fields.

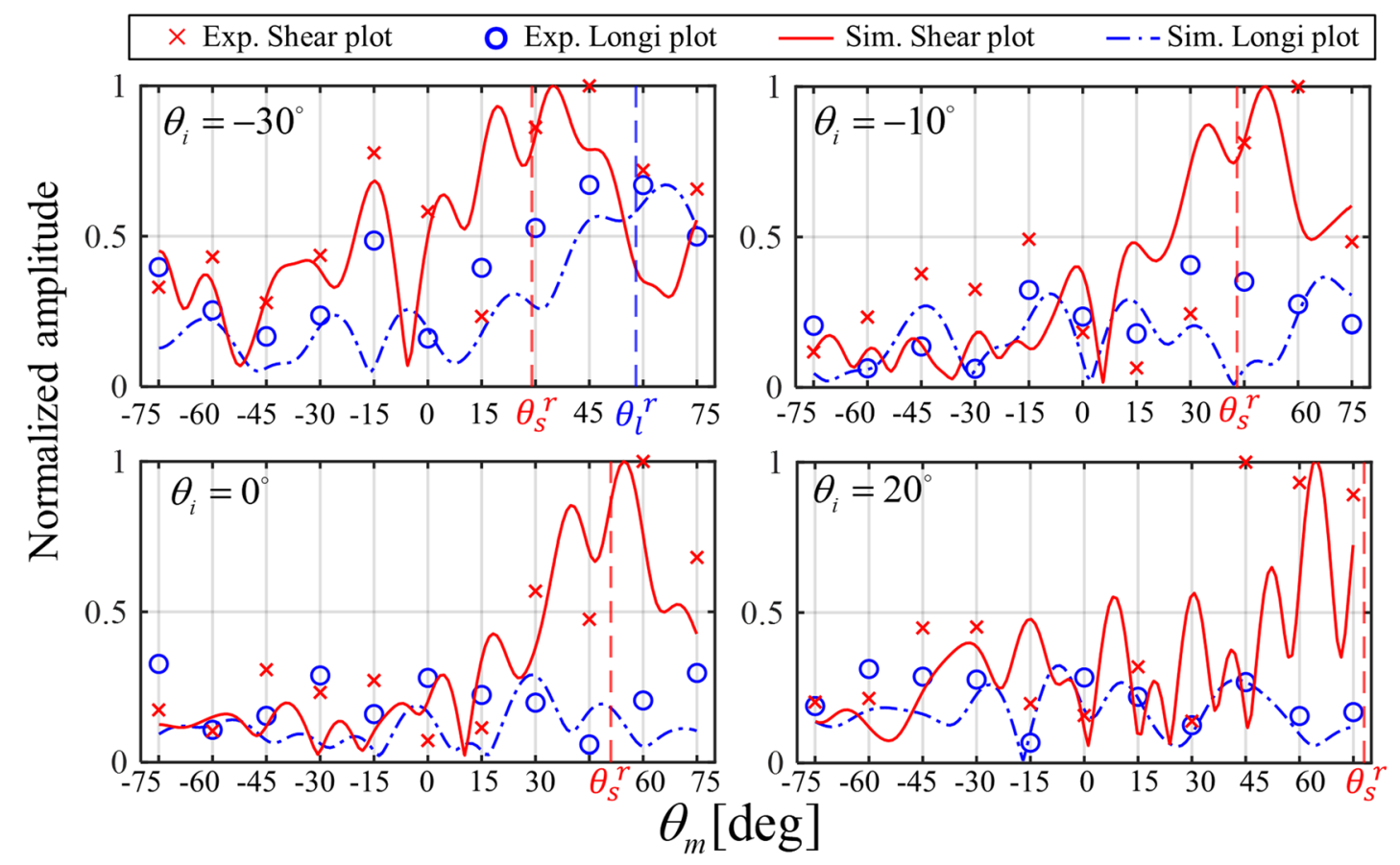

FIG. 5. Numerically and experimentally measured longitudinal and shear refracted waves. The red and blue dotted lines correspond to the numerically measured shear and longitudinal field, respectively, while the red and blue markers correspond to the experimentally measured ones. The vertical dotted line refers to the analytically calculated refraction angle. 
total mode conversion is realized for various incident angles, as expected. The detailed experimental setups are described in the supplementary material.

In conclusion, a broad angle refractive transmodal metasurface is realized by a sufficiently large phase gradient and full transmission. We utilized the single layer metasurface to overcome the difficulty of the full transmission that the Fabry-Pérot resonance cannot be applied. We designed 4 unit cells consisting of a vertical resonator and a horizontal resonator, which individually modulate the effective stiffness and mass, respectively, for impedance matching and realizing phase shifts. Numerical and experimental validations showed that our metasurface can convert the incident longitudinal wave into refracted shear wave with a broad incident angle from $-20.4^{\circ}$ to $22.3^{\circ}$. We expect that our proposed metasurface can provide a big breakthrough in various wave-based applications such as vibration tailoring, ultrasonic imaging, or NDE.

See the supplementary material for the detailed analytic procedures of deriving the transmission ratio, phase shift, and an analysis of unit cell structure for realizing effective parameters. Also the experimental setups and procedures are described.

This work was supported by the Center for Advanced MetaMaterials (CAMM) funded by the Ministry of Science, ICT and Future Planning as Global Frontier Project (No. CAMM2014M3A6B3063711), by the National Research Foundation of Korea (NRF) grants funded by the Korea Government (Nos. 2020R1A2C4002383 and 2019R1A2C1006131), by the National Research Council of Science \& Technology (NST) grant by the Korean government (MSIP; No. CAP-17-04-KRISS), and by Korea Environment Industry and Technology Institute (KEITI) through Advanced Water Management Research Program, funded by Korea Ministry of Environment (MOE) (Grant No. 127584).

\section{DATA AVAILABILITY}

The data that support the findings of this study are available from the corresponding author upon reasonable request.

\section{REFERENCES}

${ }^{1}$ K. F. Graff, Wave Motion in Elastic Solids (Dover, New York, 1975).
${ }^{2}$ J. D. Achenbach, Wave Propagation in Elastic Solids (Elsevier, New York, 1984).

${ }^{3}$ G. Clement, P. White, and K. Hynynen, J. Acoust. Soc. Am. 115, 1356 (2004).

${ }^{4}$ N. I. Saletes, T. Filleter, and A. Sinclair, NDT. E Int. 75, 72-79 (2015).

${ }^{5}$ N. Nakamura, H. Ogi, M. Hirao, and K. Nakahata, NDT. E. Int. 45(1), 156-161 (2012).

${ }^{6}$ M. Hirao and H. Ogi, NDT. E. Int. 32, 127-132 (1999).

${ }^{7}$ X. Su, Z. Lu, and A. N. Norris, J. Appl. Phys. 123, 091701 (2018).

${ }^{8}$ X. N. Liu, G. K. Hu, G. L. Huang, and C. T. Sun, Appl. Phys. Lett. 98, 251907 (2011).

${ }^{9}$ J. H. Oh, H. M. Seung, and Y. Y. Kim, Appl. Phys. Lett. 104, 073503 (2014).

${ }^{10}$ J. H. Oh, Y. E. Kwon, H. J. Lee, and Y. Y. Kim, Sci. Rep. 6, 23630 (2016).

${ }^{11}$ J. H. Oh and B. Assouar, Sci. Rep. 6, 33410 (2016).

${ }^{12}$ V. E. Gusev and O. B. Wright, New J. Phys. 16, 123053 (2014).

${ }^{13}$ S. W. Lee and J. H. Oh, Sci. Rep. 8, 14243 (2018).

${ }^{14}$ H. W. Park and J. H. Oh, Sci. Rep. 9, 13973 (2019).

${ }^{15}$ Y. Wu, Y. Lai, and Z.-Q. Zhang, Phys. Rev. Lett. 107, 105506 (2011).

${ }^{16}$ R. Zhu, X. Liu, G. Hu, C. Sun, and G. Huang, Nat. Commun. 5, 5510 (2014).

${ }^{17}$ R. Zhu, X. N. Liu, and G. L. Huang, Wave Motion 55, 73-83 (2015).

${ }^{18}$ J. M. Kweun, H. J. Lee, J. H. Oh, H. M. Seung, and Y. Y. Kim, Phys. Rev. Lett 118, 205901 (2017).

${ }^{19}$ H. J. Lee, J.-R. Lee, S. H. Moon, T.-J. Je, E.-C. Jeon, K. Kim, and Y. Y. Kim, Sci. Rep. 7, 15378 (2017).

${ }^{20}$ Y. Li, B. Liang, Z. Gu, X. Zou, and J. Cheng, Sci. Rep. 3, 2546 (2013).

${ }^{21}$ Y. Xie, W. Wang, H. Chen, A. Konneker, B. I. Popa, and S. A. Cummer, Nat. Commun. 5, 5553 (2014).

${ }^{22}$ H. Zhu and F. Semperlotti, Phys. Rev. Lett. 117, 034302 (2016).

${ }^{23}$ W. Wang, Y. Xie, B. I. Popa, and S. A. Cummer, J. Appl. Phys. 120, 195103 (2016).

${ }^{24}$ B. Liu, W. Zhao, and Y. Jiang, Sci. Rep. 6, 38314 (2016).

${ }^{25}$ K. Song, J. Kim, S. Hur, J.-H. Kwak, S.-H. Lee, and T. Kim, Sci. Rep. 6, 32300 (2016).

${ }^{26}$ Y. Liu, Z. Liang, F. Liu, O. Diba, A. Lamb, and J. Li, Phys. Rev. Lett. 119, 034301 (2017).

${ }^{27}$ H. Lee, J. K. Lee, H. M. Seung, and Y. Y. Kim, J. Mech. Phys. Solids. 112, 577-593 (2018).

${ }^{28}$ J. Rong, W. Ye, S. Zhang, and Y. Liu, Adv. Funct. Mater. 2005285 (2020).

${ }^{29}$ L. Cao, Z. Yang, Y. Xu, S. W. Fan, Y. Zhu, Z. Chen, Y. Li, and B. Assouar, J. Mech. Phys. Solids 143, 104052 (2020).

${ }^{30}$ J. Zhang, X. Zhang, F. Xu, X. Ding, M. Deng, N. Hu, and C. Zhang, J. Sound. Vib 481, 115440 (2020).

${ }^{31}$ L. Cao, Z. Yang, Y. Xu, S. W. Fan, Y. Zhu, Z. Chen, B. Vincent, and B. Assouar, Phys. Rev. Appl. 13, 014054 (2020).

${ }^{32}$ M. S. Kim, W. R. Lee, Y. Y. Kim, and J. H. Oh, Appl. Phys. Lett 112, 241905 (2018).

${ }^{33}$ S. W. Lee and J. H. Oh, J. Phys. D: Appl. Phys 53, 265301 (2020).

${ }^{34}$ N. Yu, P. Genevet, M. Kats, F. Aieta, J. Tetienne, F. Capasso, and Z. Gaburro, Science 334, 333 (2011). 